\title{
Molecular Structure of Brown-Dwarf Disks
}

\author{
D. S. Wiebe ${ }^{1}$, D. A. Semenov ${ }^{2}$, and T. Henning ${ }^{2}$ \\ ${ }^{1}$ Institute of Astronomy of Russian Academy of Sciences, Moscow, Russia \\ ${ }^{2}$ Max Planck Institute for Astronomy, Heidelberg, Germany \\ Received November 25, 2007; in final form, April 11, 2008
}

\begin{abstract}
We describe typical features of the chemical composition of proto-planetary disks around brown dwarfs. We model the chemical evolution in the disks around a low-mass T Tauri star and a cooler brown dwarf over a time span of 1 Myr using a model for the physical structure of an accretion disk with a vertical temperature gradient and an extensive set of gas-phase chemical reactions. We find that the disks of T Tauri stars are, in general, hotter and denser than the disks of lower-luminosity substellar objects. In addition, they have more pronounced vertical temperature gradients. The atmospheres of the disks around low-mass stars are more strongly ionized by UV and X-ray radiation, while less dense brown-dwarf disks have higher fractional ionizations in their midplanes. Nevertheless, in both cases, most molecules are concentrated in the so-called warm molecular layer between the ionized atmosphere and cold midplane, where grains with ice mantles are abundant.
\end{abstract}

PACS numbers: 97.21.+a, 97.20.Vs, 97.10.Tk

DOI: $10.1134 / \mathrm{S} 1063772908110103$

\section{INTRODUCTION}

Brown dwarfs represent the "missing link" between bodies of stellar and planetary mass. A brown dwarf (or substellar object) is defined to be a body with a mass that is not high enough to support longlasting thermonuclear reactions converting hydrogen into helium, but is sufficiently high to enable a short stage when thermonuclear reactions involving deuterium can occur. In our current picture of stellar evolution, this means that the masses of brown dwarfs are approximately $13-80$ Jovian masses $\left(M_{\mathrm{J}}\right)[1]$.

Moreover, the intermediate status of brown dwarfs is emphasized by the fact that it remains unclear whether they are closer genetically to planets or stars. Both "planet-like" and "star-like" scenarios for their formation have been discussed [2]. In the former scenarios, brown dwarfs form in proto-planetary disks via the same processes that result in the formation of planets, and later leave the system due to gravitational interactions. The latter scenarios suppose that there is a single process that produces brown dwarfs and stars, at least low-mass stars.

There are several different star-like scenarios for the formation of brown dwarfs. First, the evolutionary histories of stellar and substellar objects could be completely identical. In this case, the transformation of a given prestellar core into a low-mass star or a brown dwarf depends only on the mass and angular momentum of the core. Second, a low-mass protostar could be transformed into a proto-brown-dwarf due to the action of external factors that abort its growth. The mass of a prestellar core could decrease due to its evaporation by UV radiation from nearby stars [3-5]. The accretion of matter onto a prestellar core could be terminated due to its gravitational interaction with other cores or young stars, resulting in its ejection from the region of the associated gas-rich molecular cloud [6]. Of course, all these processes could operate in Nature; however, recent data on the mass function of brown dwarfs and the structure of matter surrounding them provide evidence that most brown dwarfs are formed via the same processes that lead to the formation of low-mass stars.

One of the arguments in favor of a single evolutionary scenario for stellar and substellar objects comes from observations of accretion disks around brown dwarfs. In terms of their parameters (lifetime, relative occurrence), these disks seem to be similar to the disks of T Tauri stars, and obey the dependence of the accretion rate on the mass of the central object for T Tauri stars, $\dot{M} \propto M^{2}$ [7]. However, there remains a large scatter in the disk masses, due to uncertainties in the parameters of the associated dust. Moreover, estimation of the disk masses requires observations in the millimeter and submillimeter, but such observations are still relatively rare. The first observations of this kind were carried out by Klein et al. [8], whose JCMT and IRAM observations led to the discovery of long-wavelength dust emission from the disks of the brown dwarfs CFHT-BD-Tau 4 and IC 348 613. They derived the mass estimates 
$(0.4-2.4) M_{\mathrm{J}}$ and $(1.7-5.7) M_{\mathrm{J}}$, respectively. Similar masses (from one-tenth to several $M_{\mathrm{J}}$ ) were also obtained for the disks of other brown dwarfs (see, e.g., [9]). These data differ from the data for the disks of T Tauri stars, which usually have $0.01-0.1 M_{\odot}$, independent of the mass of the central object.

There is currently no consensus about the structure of the disks. Natta and Testi [10] concluded that the IR spectra of brown-dwarf disks can be reproduced by the same flared-disk models as the spectra of $\mathrm{T}$ Tauri stars. However, later studies (see [11] and references therein) have shown that a considerable fraction of these disks may be flatter, due to the growth of grains and their settling to the midplane of the disk [12]. On the other hand, the theoretical model presented, for instance, by Walker et al. [13] shows that the disks of brown dwarfs may be more flared than circumstellar disks. More detailed studies of brown-dwarf disks will require molecular, atomic, and ionic spectral-line observations with high spatial and spectral resolution.

Due to the low masses of brown-dwarf disks, observations require detectors with high sensitivities and long accumulation times. Some vibrational or vibrational-rotational lines are accessible by the IRS spectrograph of the $S$ pitzer space telescope and the IRAM interferometer (Plateau de Bure, France). It will become possible to observe other lines with future detectors, such as the ALMA radio interferometer and the Herschel space observatory. The aim of our present study is to model the chemical structures of brown-dwarf disks and compare them with the structures of proto-planetary disks of $\mathrm{T}$ Tauri stars. Section 2 describes the models for the structure and chemical evolution of proto-planetary disks adopted here. The modeling results are presented in Section 3, and discussed in Section 4. Section 5 presents our conclusions.

\section{MODEL FOR THE STRUCTURE AND CHEMICAL EVOLUTION OF THE DISK}

The basis for modeling the accretion disks of young stars in many studies is the so called "alphadisk" suggested by Shakura and Syunyaev [14]. This model is based on the assumption that the transfer of angular momentum in the disk is caused by turbulent viscosity, with the viscosity coefficient $\nu$ written $\alpha H c_{\mathrm{s}}$, where $H$ is the disk semi-thickness at a given annulus, $c_{\mathrm{S}}$ is the isothermal sound speed, and $\alpha$ is a parameter that is approximately $0.001-0.1$ for models of proto-planetary disks (see the discussion of this model in [15]).

We have implemented a simplified version of the model described by D'Alessio et al. [16, 17]. The essence of this simplification is that, in contrast to
[16, 17], we include only two sources of heating when computing the gas temperature (which we must know to integrate the equation of hydrostatic equilibrium): viscous dissipation and irradiation of the disk by the central object. A comparison of our model disks with the models of D'Alessio et al. [16, 17] shows that this simplification does not lead to differences in the disk structure that are important for modeling their chemical structure.

In the model we used, the disk structure is described by the equations

$$
\begin{gathered}
\frac{d P}{d z}=-g \rho, \\
\frac{d T}{d z}=-\frac{3 \kappa \rho F}{4 a c T^{3}}, \\
\frac{d F}{d z}=\frac{9}{4} \alpha P \Omega(r)+\Gamma_{\text {irr }} .
\end{gathered}
$$

Here, $P$ is the gas pressure, $g$ the gravitational acceleration, $\rho$ the gas density, $T$ the gas temperature, $F$ the disk-radiation flux, $\Omega(r)$ the angular velocity of the disk at a distance $r$ from the central object, and $\Gamma_{\text {irr }}$ the rate of heating of the disk by radiation from the central object. We modeled the two-dimensional structure of the disk in the so-called 1+1D approximation, which assumes that the time scale for motions in the vertical direction is much shorter than the time scale for radial motions. For this reason, the disk is in hydrostatic equilibrium [defined by (1)] at all $r$. As a result, we can find the vertical structure of the disk by solving the system (1)-(3) independently for each $r$. To compute the temperature in each vertical bin, we integrate the radiative transfer equation (2) in the Eddington approximation. The first term in (3) describes viscous heating of the disk material. In this formulation of the problem, the disk is described by the

- accretion rate $\dot{M}$;

- viscosity parameter $\alpha$;

- temperature $T_{*}$, radius $R_{*}$, and mass $M_{*}$ of the central object (which is assumed to be a blackbody);

- inner and outer radii of the disk $R_{\text {in }}$ and $R_{\text {out }}$.

We considered four disk models with the parameters given in the Table. Model T Tauri corresponds to the disk of a young $\mathrm{T}$ Tauri star whose parameters correspond to those of DM Tau [18]. Models with the prefix BD correspond to the disk of a brown dwarf with a mass of $0.06 M_{\odot}$. The remaining parameters were taken from [19], assuming that the age of the dwarf is 
Models of the structure of proto-planetary disks

\begin{tabular}{l|c|c|c|c|c|c}
\hline \multicolumn{1}{c|}{ Model } & $\dot{M}, M_{\odot} / \mathrm{yr}$ & $\alpha$ & $M_{*}, M_{\odot}$ & $R_{*}, R_{\odot}$ & $T_{*}, \mathrm{~K}$ & Disk mass, $M_{\mathrm{J}}$ \\
\hline T Tauri & $2 \times 10^{-9}$ & 0.01 & 0.65 & 1.2 & 3720 & 30 \\
BD2e-9 & $2 \times 10^{-9}$ & 0.01 & 0.06 & 0.7 & 2900 & $4.7(r<300 \mathrm{AU})$ \\
BD1e-12 & $10^{-12}$ & 0.01 & 0.06 & 0.7 & 2900 & $3.0(r<300 \mathrm{AU})$ \\
BD1e-12 $\alpha$ & $10^{-12}$ & 0.1 & 0.06 & 0.7 & 2900 & $2.2(r<300 \mathrm{AU})$ \\
\hline
\end{tabular}

1 Myr. Model BD2e-9 differs from model T Tauri only in the parameters of the central object. The accretion

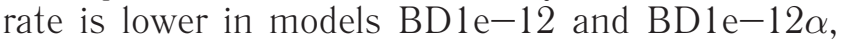
and the value of $\alpha$ is increased in model BD $1 \mathrm{e}-12 \alpha$.

The last row of the Table gives the total mass of the disk. Our modeling of the disk structure assumed that $R_{\text {in }}=0.03 \mathrm{AU}$ and $R_{\text {out }}=800$ AU. No definite information about the outer radii of brown-dwarf disks is available, but it is believed that these disks are smaller than circumstellar proto-planetary disks. Accordingly, we considered only regions with $r<$ $300 \mathrm{AU}$ when calculating the masses of the BD disks. Figure 1 shows the main parameters of the disks we consider here-surface density, semi-thickness, density, and temperature in the equatorial plane-as functions of radius. We can see that the disks of lowmass stars are hotter, denser, and thinner than the disks of brown dwarf. In the models with high accretion rates ( T Tauri and BD2e-9), the surface density drops substantially with radius (by three orders of magnitude), while it is almost independent of radius in the models with low accretion rates (BD1 e-12 and BD $1 \mathrm{e}-12 \alpha$ ). Figure 2 presents the vertical distributions of the temperature and density at $r=1 \mathrm{AU}$. Here, also, we can see that the disks of low-mass stars are thinner, in agreement with the computations of Walker et al. [13].

The density and temperature distributions computed using the model for the disk structure were applied further to compute the parameters needed to model the chemical evolution: the relative intensity of the UV radiation and the rate of ionization by cosmic rays and $\mathrm{X}$-rays. This part of the model is described in detail in [20]. In the present study, we assumed that the unattenuated intensity of the stellar UV emission at a distance of $100 \mathrm{AU}$ in the T Tauri model exceeds the standard interstellar radiation field by a factor of 540 [21]. The BD models do not include UV radiation of the star. The mean Galactic UV background is included in all the models. The X-ray luminosity of the central object is specified to be $10^{30} \mathrm{erg} / \mathrm{s}$ in the $\mathrm{T}$ Tauri model and $10^{28} \mathrm{erg} / \mathrm{s}$ in the BD models [13].

The chemical-kinetics equations were integrated without taking into account surface reactions, but allowing for the formation and destruction of the ice mantles of dust grains. We used the chemicalreaction data from the UMIST95 database [23]. Altogether, the model takes into account about 500 gas-phase and surface components that are involved in 5000 reactions. As initial abundances, we used the low-metallicity atomic abundances from [24].

\section{RESULTS}

In this Section, we describe the key differences in the molecular compositions in the models considered at an age of 1 Myr. We consider distances from 1100 AU only. The differences between the models are insignificant for $r>100$ AU (Fig. 1), while the temperature and density of the disk are so high at $r<$ $1 \mathrm{AU}$ that effects associated with the vaporization of dust grains and three-body reactions that were not taken into account start to play an important role. Naturally, the differences in the molecular composition reflect differences in the physical conditions in the disks considered, which can be summarized as follows. The disks of $\mathrm{T}$ Tauri stars have a higher temperature (especially, close to the surface) and density. A strong dissociation effect due to the stellar radiation is present in the surface layers. On the other hand, due to the high density of the central zone, the region influenced by the $\mathrm{X}$-ray radiation of the hot stellar corona is small. The disk in model BD2e-9 is slightly less dense, but the role of $\mathrm{X}$-ray radiation in the formation of its molecular composition is also insignificant. Of course, this is related, to a considerable extent, to the fact that the $\mathrm{X}$-ray luminosity of a brown dwarf is two orders of magnitude lower than that of a T Tauri star. It is important that, in the BD2e-9 disk model, the difference between the temperatures in the equatorial plane of the disk and at its surface is less than for the disk of a T Tauri star. The reason for this is the much lower luminosity and effective temperature of a brown dwarf as compared to a T Tauri star (Fig. 2).

Finally, the surface density of the disk in model BD $1 \mathrm{e}-12$ is low and varies weakly with distance. Therefore, in this model, the disk is more transparent to both X-ray radiation, which is incident predominantly in the radial direction, and cosmic rays, which penetrate the disk from above. Moreover, in this model, the thickest surface layer is the one heated 

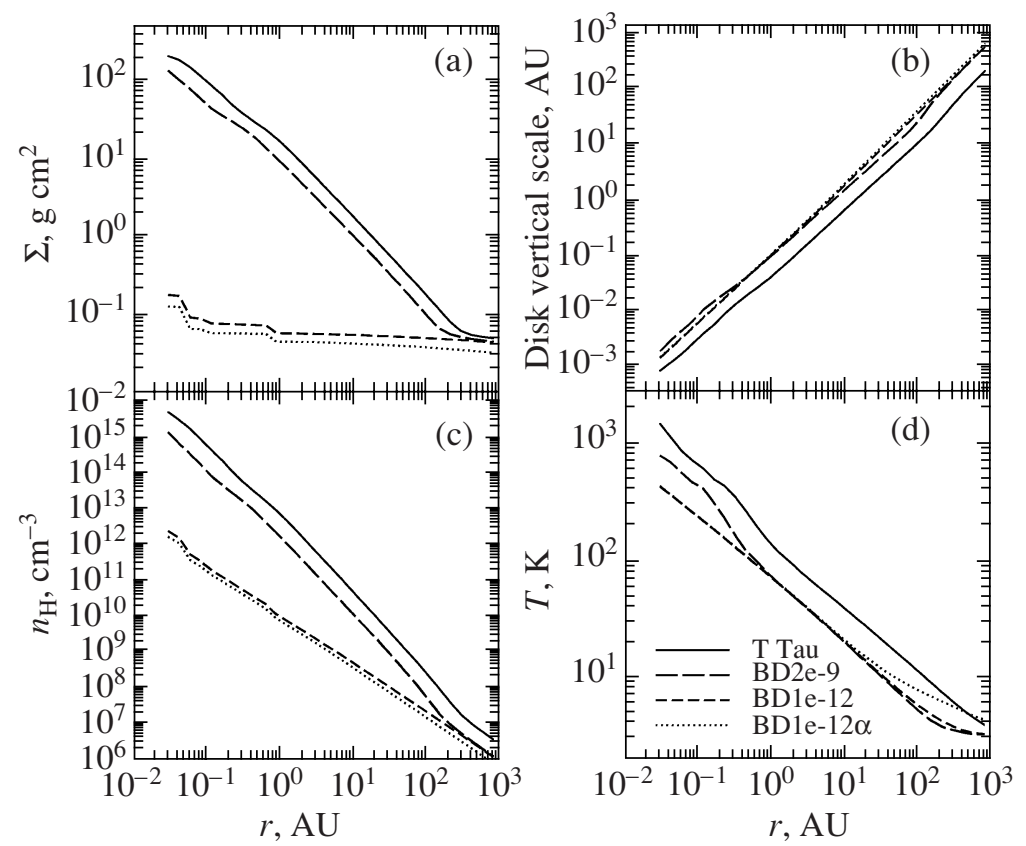

Fig. 1. Radial distribution of the main disk parameters: (a) gas surface density, (b) vertical scale of the gas distribution, (c) density, and (d) temperature in the disk midplane.
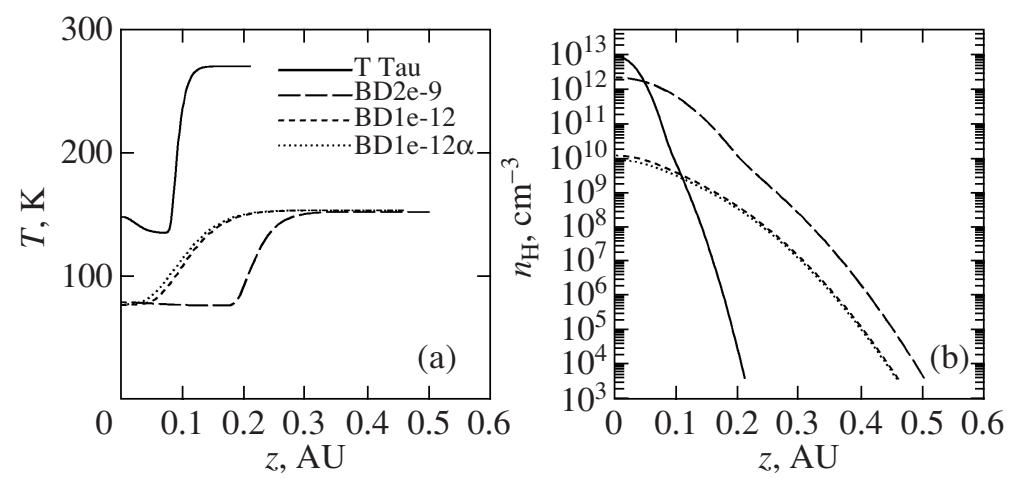

Fig. 2. Vertical distribution of the gas temperature and density of the disk at a distance of $1 \mathrm{AU}$ from the central object (star or brown dwarf).

by the radiation of the central object. Overall, this results in a much higher degree of ionization $x_{\mathrm{e}}$ in disk model BD1e-12. While $x_{\mathrm{e}}$ does not exceed $10^{-13}$ in more massive disks, the electron concentration in model BDe-12 does not decrease below $10^{-10}$. Magneto-rotational instability is thought to be one of the main effects of the excitation of turbulence in proto-planetary disks, and the degree of ionization, which determines the efficiency of the interaction between the matter and magnetic field, could turn out to be a critical parameter in more detailed disk models.

Figure 3 compares the degree of ionization in three models. In addition to the higher ionization in model BD $1 \mathrm{e}-12$ noted above (bottom diagram), another interesting peculiarity is related to the region of minimum ionization in models T Tauri and BG2e9 , which is located appreciably farther from the central object in the circumstellar-disk model (upper diagram) than in the brown-dwarf disk model. This is due to the fact that there remains magnesium in the gas phase at $r<(3-4)$ AU in the T Tauri disk, whose ions enable degrees of ionization of $\sim 10^{-9}$, even in a cool (relative to the atmosphere) disk midplane. In the cooler BD2e-9 disk and the T Tauri disk at $r<(3-4)$ AU, magnesium is frozen out onto dust grains, and the dominant ion is the less abundant $\mathrm{HCO}^{+}$. The dominant ion in the main part of the $\mathrm{BD} 1 \mathrm{e}-12$ disk is $\mathrm{H}_{3}^{+}$; this is a direct consequence of the enhanced rate of ionization by $\mathrm{X}$-rays and cosmic rays. 


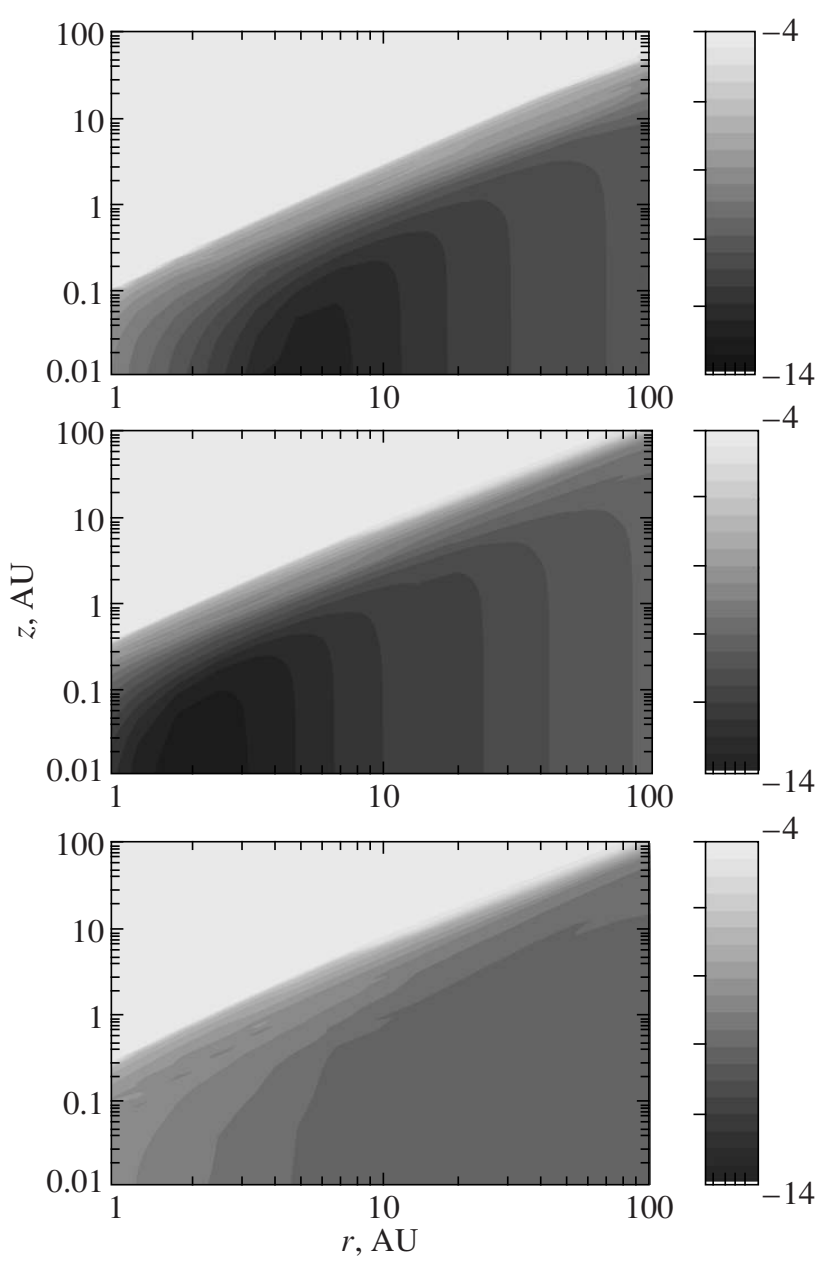

Fig. 3. Degree of ionization in models T Tauri (top), BD2e-9 (middle), and BD1e-12 (bottom).

In our simple formulation of the problem, we can take into account the enhanced accretion efficiency in disks with higher degrees of ionization by assuming a larger value of $\alpha$. As we can see in the Table, the disk in the $\alpha=0.1$ model has the lowest mass among all the models considered, indicating more efficient accretion. However, the differences in molecular composition between models BD1e12 and BD1 $-12 \alpha$ are insignificant, and we do not consider model BD $1 \mathrm{e}-12 \alpha$ further.

\subsection{Oxygen- and Nitrogen-Bearing Compounds}

A typical feature of all our models is the presence of a so-called warm molecular layer [25], where the temperature is already so high that most molecular components do not freeze out onto the surfaces of grains, but the intensity of the UV radiation remains insufficient for the efficient dissociation of molecules. In the T Tauri and BD2e-9 models, there is a zone of reduced gas-phase abundance of most molecules below the warm molecular layer. At the distance $r>$
$50 \mathrm{AU}$ in the T Tauri model and $r>(10-20) \mathrm{AU}$ in model BD2 $\mathrm{e}-9$, this zone totally encompasses the central region of the disk; its origin is related to the formation of ice mantles of the grains. Closer to the central object, the picture is more complex. To illuminate this, Figure 4 shows the distribution of molecular nitrogen; other simple molecules, such as $\mathrm{CO}$ and $\mathrm{O}_{2}$, have similar distributions.

The $\mathrm{N}_{2}$ abundance drops sharply with decreasing $z$ in the inner region of the disk immediately beneath the warm molecular layer, then increases, with molecular nitrogen again becoming the dominant nitrogen compound in the equatorial plane. In the narrow region of reduced $\mathrm{N}_{2}$ abundance, virtually all nitrogen atoms are bound in ammonia molecules in grain mantles. Other major constituents of the mantles are water molecules, which bind most of the oxygen atoms. This is the reason for the reduction of the $\mathrm{CO}$ and $\mathrm{O}_{2}$ abundances in the gas-phase at intermediate $z$, while oxygen atoms are again found in the gas phase in the middle region of the disk, either free or bound in $\mathrm{CO}$ molecules. The abundance 

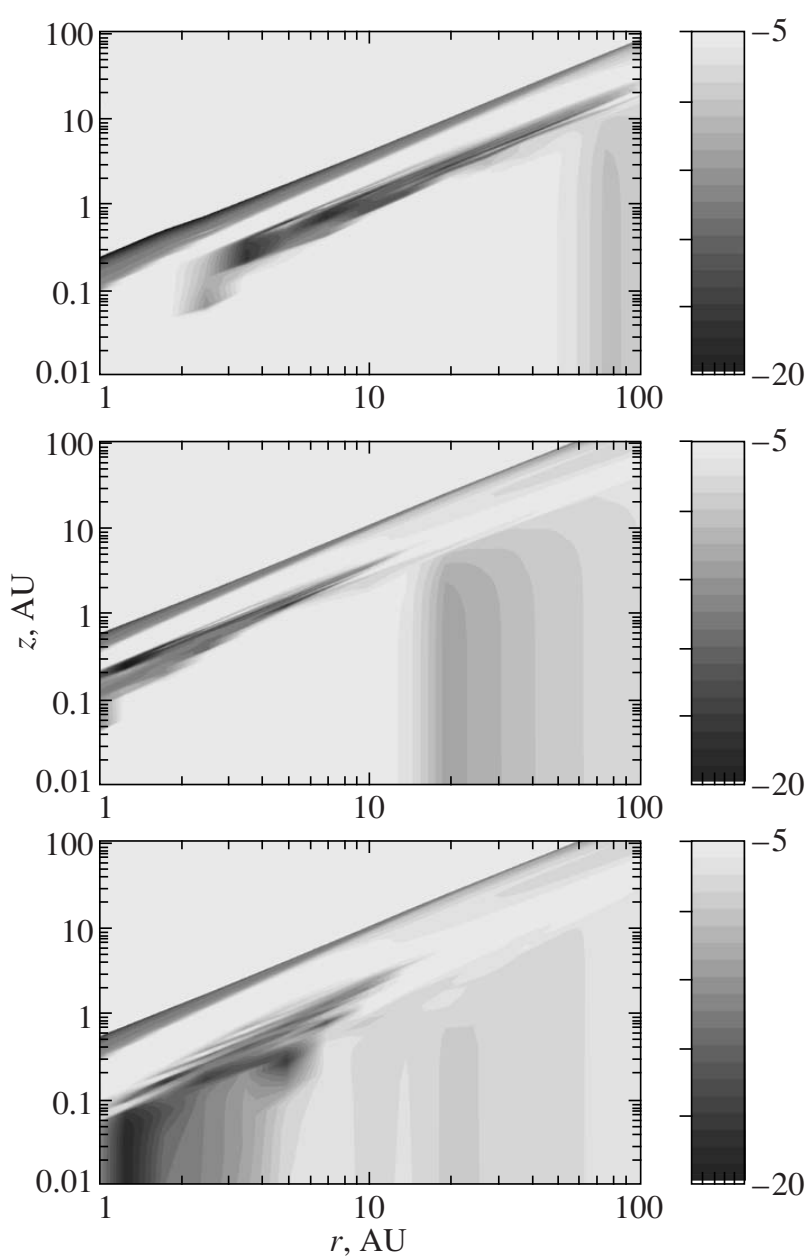

Fig. 4. Same as Fig. 3 for the abundance of $\mathrm{N}_{2}$.

of water ice on grains also grows with distance from the disk, but this does not influence the gas-phase abundance of $\mathrm{CO}$, since this abundance in the central region of the disk is determined by the number of $\mathrm{C}$ atoms specified in the model.

The reason for the formation of a narrow layer of frozen-out molecules (we will call this the mantle zone) at small $r$ in the T Tauri and BD2e-9 models is the thermal structure of the disk. At $r \sim 10 \mathrm{AU}$, the modest gas temperature in the equatorial plane (20 K in BD2e-9 and $40 \mathrm{~K}$ in $\mathrm{T}$ Tauri) inhibits the efficient synthesis of hydrogen-saturated water and ammonia molecules in the gas phase. However, the temperature rises with increasing $z$, enhancing the rate of formation of $\mathrm{H}_{2} \mathrm{O}$ and $\mathrm{NH}_{3}$ molecules. Molecules formed in the gas phase freeze out onto the surfaces of grains. It is important here that the temperature in a certain height range remains insufficiently high to totally vaporize the ice mantles, since the desorption energies of $\mathrm{H}_{2} \mathrm{O}$ and $\mathrm{NH}_{3}$ are quite high. As a result, a layer is formed where ammonia and water are synthesized and freeze out onto grains, representing, in essence, an irreversible sink of nitrogen and oxygen atoms and resulting in a sharp decrease of the $\mathrm{CO}$ and $\mathrm{N}_{2}$ abundances. In higher and hotter regions of the disk, the higher $\mathrm{H}_{2} \mathrm{O}$ and $\mathrm{NH}_{3}$ abundances hinder photodissociation of these molecules. The rate of photodissociation of $\mathrm{N}_{2}$ in this zone is almost an order of magnitude lower than the rate of photodissociation of ammonia. The rate of photodissociation of $\mathrm{CO}$ is also appreciably lower than the rate of photodissociation of water, and is further reduced by screening by $\mathrm{H}_{2}$ molecules, which are dissociated by photons with approximately the same wavelength as those dissociating the CO.

In contrast to the massive-disk models, a significant abundance of simple nitrogen and oxygen compounds in the gas phase at low heights is not observed in model BD1 e-12. Over the entire equatorial region of the disk, most nitrogen atoms are bound in ice mantles, primarily in $\mathrm{HCN}, \mathrm{NH}_{3}$, and $\mathrm{NO}$. Grain ice mantles, or more precisely, the $\mathrm{H}_{2} \mathrm{O}, \mathrm{CO}$, and $\mathrm{O}_{2}$ molecules they contain, serve as the main reservoir for oxygen atoms in this model. 


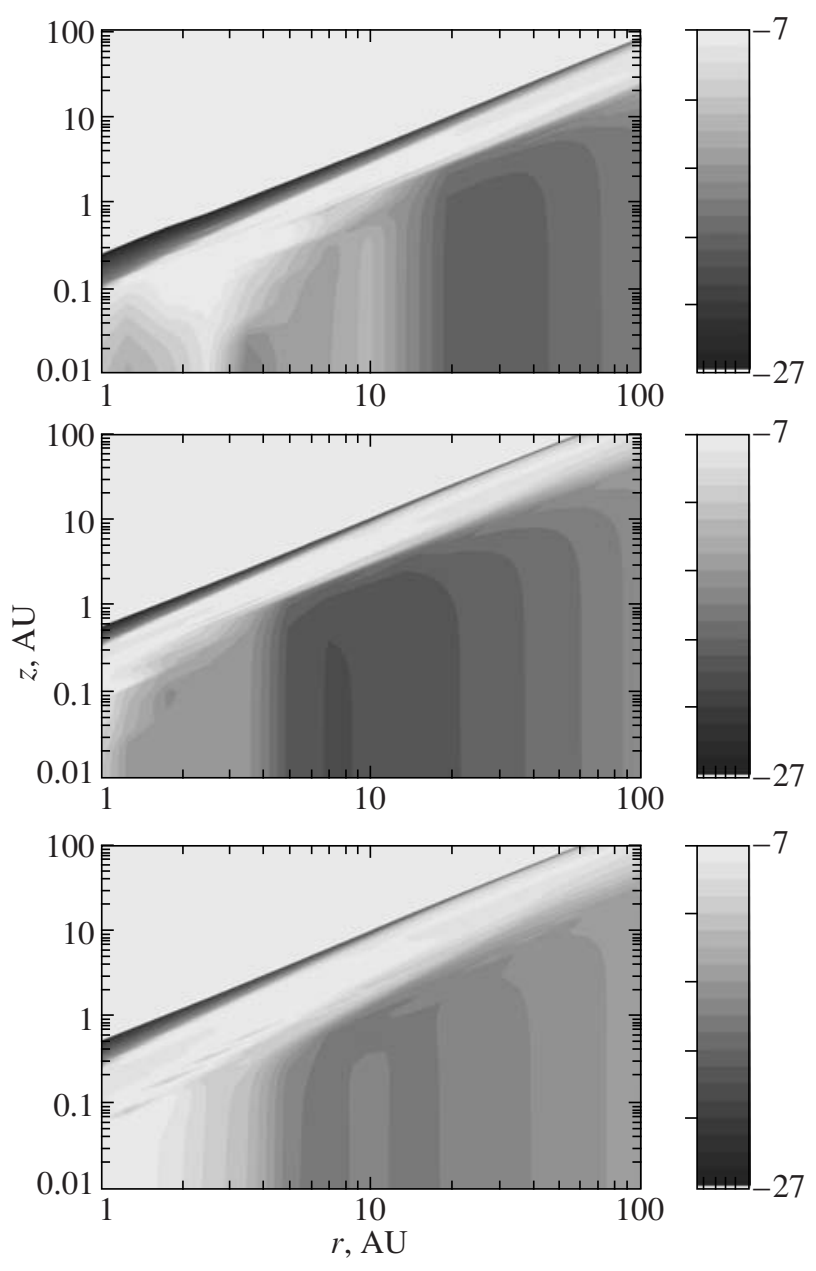

Fig. 5. Same as Fig. 3 for CS.

\subsection{Carbon-Bearing Compounds}

In general, the distribution of carbon compounds shows the same pattern as the distributions of nitrogen and oxygen compounds: there is a mantle zone just beneath the warm molecular layer; i.e., a zone where grain ice mantles form especially efficiently, leading due to a combination of physical factors to carbon atoms "sinking" into molecules with high desorption energies. In molecular clouds and prestellar cores, carbon atoms are bound predominantly in CO molecules, which have a high dissociation potential. However, in the cool, relatively dense layer considered, the initial binding of carbon atoms in $\mathrm{CO}$ molecules is suppressed by ion-molecular reactions that result in the formation of water molecules, which later freeze out. Due to this process, oxygen atoms become deficient in the gas phase, and the synthesis of more complex carbon compounds-carbon chains and cyanopolynes - becomes possible. Since the desorption energies of these compounds are high, they also freeze out onto the dust grain surfaces.
In the T Tauri model, at $r=5 \mathrm{AU}$ and heights from 0.3 to $0.65 \mathrm{AU}, \mathrm{HCN}$ in the grain phase (i.e., in grain ice mantles) becomes the dominant carbon compound. Molecules such as $\mathrm{HC}_{3} \mathrm{~N}, \mathrm{C}_{5} \mathrm{H}, \mathrm{C}_{7}$, and even $\mathrm{C}_{8} \mathrm{H}$, also in the grain phase, are only slightly less abundant. In model BD2e-9 (also at $r=5 \mathrm{AU}$ ), the zone of carbon dominance in the grain phase is larger, and extends from $z=1 \mathrm{AU}$ to $z=2$ AU. However, the process of synthesis goes as far as forming complex carbon chains only in its upper region. The chemical composition in the lower region of the mantle zone is less exotic, and carbon atoms are bound in surface molecules of acetylene and formaldehyde. In model BD1e-12, the mantle zone extends to the disk midplane; i.e., in contrast to models $\mathrm{T}$ Tauri and BD2e-9, it does not form a layer. In these models, the process of synthesis in the lower part of the mantle zone over 1 Myr proceeds only as far as the formation of acetylene and carbon dioxide. 

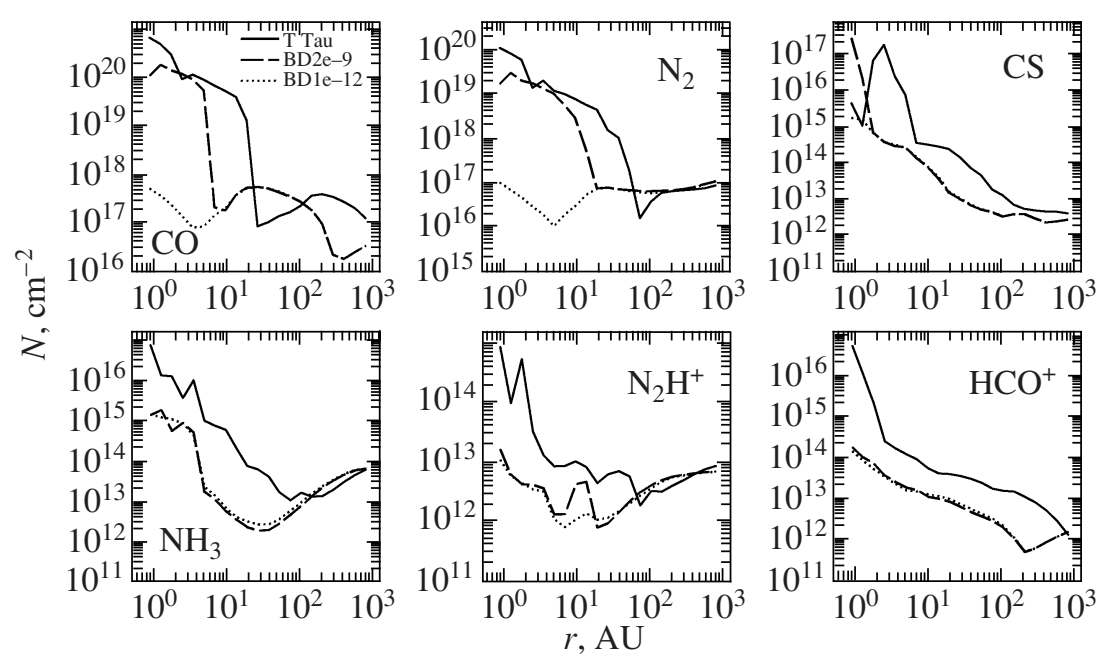

Fig. 6. Distributions of some molecules in our models. The column density is shown along the vertical axis.

\subsection{Sulfur-Bearing Compounds}

Figure 5 shows the distribution of CS molecules over the disk. In all three models, the CS molecules at $r>(7-8)$ AU are distributed in a warm molecular layer. At lower distances, the CS distributions are considerably different. With approach to the center of the disk in the T Tauri model, the region of enhanced CS abundance first expands, approaching the disk symmetry plane at $r \approx 2.5$ AU. However, with further decrease in $r$, the CS abundance at $z<$ 0.1 AU decreases: the high temperatures in this region activate neutral-neutral reactions that lead predominantly to the formation of OCS, SO, and $\mathrm{SO}_{2}$ $\left(\mathrm{CS} \rightarrow \mathrm{OCS} \rightarrow \mathrm{SO} \rightarrow \mathrm{SO}_{2}\right.$ ). At somewhat larger distances from the center $(r \sim 5 \mathrm{AU})$, sulfur atoms are also mainly bound in these molecules, but now in the grain rather than the gas phase. In the cooler disk of model BD2e-9, the rate of OCS, SO, and $\mathrm{SO}_{2}$ synthesis is not as high, but the rate at which these molecules freeze out onto dust grains increases. As a result, the reservoir of $\mathrm{S}$ atoms in the entire inner region of the disk become bound in OCS and $\mathrm{SO}_{2}$ molecules in the grain phase.

In model $\mathrm{BD} 1 \mathrm{e}-12$, the central part of the region of the disk is not hot enough for the efficient synthesis of OCS, SO, and $\mathrm{SO}_{2}$, and CS is the main sulfurbearing component. With increasing distance from the disk center (at $r \sim 5 \mathrm{AU}$ ) in the equatorial plane of model BD1e-12, the carbon chains $\mathrm{C}_{3} \mathrm{~S}$ and $\mathrm{C}_{4} \mathrm{~S}$ compete in abundance with simple sulfur-containing molecules, because of the deficit of oxygen atoms due to their locking in water ice on grain surfaces.

The main sulfur compounds in the warm molecular layer in all three models at $r \sim 1 \mathrm{AU}$ are $\mathrm{SO}$ and $\mathrm{SO}_{2}$. However, the region where these molecules dominate in the BD models extends to $r \sim 5 \mathrm{AU}$ only. At larger distances, CS again becomes the main sulfur compound in the warm molecular layer. Due to the relatively low desorption energy of $\mathrm{CS}$, none of the models has a mantle zone in the distribution of this molecule.

\section{DISCUSSION}

In general, the global molecular structure of the disks considered here is defined, first, by the presence of molecules with relatively low desorption energies (CO, $\mathrm{N}_{2}, \mathrm{CS}$ ) and, second, by the presence of molecules whose synthesis efficiency increases with temperature $\left(\mathrm{H}_{2} \mathrm{O}, \mathrm{NH}_{3}, \mathrm{SO}, \mathrm{SO}_{2}\right)$ and by the high desorption energies of these molecules. Photodissociation reactions that destroy predominantly $\mathrm{H}_{2} \mathrm{O}$, $\mathrm{NH}_{3}, \mathrm{SO}$, and $\mathrm{SO}_{2}$ also act in the warm molecular layer. Thus, three options are realized for these molecules in the disk:

- the temperature is high enough for both their synthesis and retention in the gas phase (the warm molecular layer);

- the temperature is high enough for their synthesis but not for their retention in the gas phase (the mantle zone);

- the temperature is too low for their synthesis.

Because of the relatively low temperature of this last region, molecules with low desorption energies are abundant there.

Figure 6 compares the column-density distributions for some of the main species. The compounds shown can tentatively be separated into two groups. The column densities of the molecules with low desorption temperatures ( $\left.\mathrm{CO}, \mathrm{N}_{2}, \mathrm{CS}\right)$ shown in the upper row of panels behave almost the same in the 
T Tauri and BD2e-9 models, except that the sharp rise of the column density associated with the emergence of molecules in the gas phase in the equatorial region of the disk starts further from the center in model $\mathrm{T}$ Tauri than in model BD2e-9. On the contrary, for molecules with large desorption energies and ions (lower row of panels in Fig. 6), models BD2e-9 and $B D 1 e-12$ are more similar (these two models are practically indistinguishable for $\mathrm{HCO}^{+}$). This shows that these molecules are concentrated almost exclusively in a warm molecular layer that is close to the surface, so that the differences in the structure of the deeper layers of the disk do not influence the distribution of the molecules. The higher column densities of molecules in the T Tauri model are determined by the heating of the disk surface by the stellar radiation.

It is important that the differences between these models are manifest over scales of the order of $10 \mathrm{AU}$, making interferometric observations necessary for their detection. More distant regions of the disks seem to be very similar in terms of the distributions of most molecules, but more definite conclusions require detailed radiative-transfer modeling.

\section{CONCLUSION}

We have used a $1+1 \mathrm{D}$ approximation to compare the structures of the disks of low-mass stars and brown dwarfs and investigate differences in the molecular compositions of the disks. These differences are associated mainly with the thermal structure of the disks, and depend substantially on the desorption energies of the molecules involved. Due to the action of various factors, apart from the warm molecular layer, molecules with relatively low desorption energies $\left(\mathrm{N}_{2}, \mathrm{O}_{2}, \mathrm{CO}\right)$ are also located in the disk equatorial plane at small distances from the center of the disk. The binding of atoms into compounds with large desorption energies results in the formation of another layer (mantle zone) just beneath the warm molecular layer, where the abundances of some molecules are substantially reduced. Possible observational manifestations of these differences will be considered in a separate paper.

\section{ACKNOWLEDGMENTS}

This work was supported by a President of the Russian Federation Grant to Support Young Doctors of Science (DSW, project code MD-4815.2006.2) and the Program of State Support of Leading Scientific Schools of the Russian Federation (project no. NSh-4354.2008.2).

\section{REFERENCES}

1. B. R. Oppenheimer, S. R. Kulkarni, and J. R. Stauffer, in Protostars and Planets IV, Ed. by V. Mannings, A. P. Boss, and S. S. Russell (Univ. Arizona Press, Tucson, 2000), p. 1313.

2. A. Whitworth, M. R. Bate, A. Nordlund, et al., in Protostars and Planets IV, Ed. by V. Mannings, A. P. Boss, S. S. Russell (Univ. Arizona Press, Tucson, 2000), p. 459.

3. D. S. Vibe, V. I. Shematovich, and B. M. Shustov, Astron. Zh. 73, 702 (1996) [Astron. Rep. 40, 639 (1996)].

4. V. I. Shematovich, D. S. Vibe, and B. M. Shustov, Astron. Zh. 76, 738 (1999) [Aston. Rep. 43, 645 (1999)].

5. A. P. Whitworth and H. Zinnecker, Astron. Astrophys. 427, 299 (2004).

6. B. Reipurth and C. Clarke, Astron. J. 122, 432 (2001).

7. J. Muzerolle, K. L. Luhman, C. Briceno, et al., Astrophys. J. 625, 906 (2005).

8. R. Klein, D. Apai, I. Pascucci, et al., Astrophys. J. Lett. 593, L57 (2003).

9. A. Scholz, R. Jayawardhana, and K. Wood, Astrophys. J. 645, 1498 (2006).

10. A. Natta and L. Testi, Astron. Astrophys. 376, 22 (2001).

11. D. Apai, K. Luhman, and M. C. Liu, e-Print arXiv: astro-ph/0702286 (2007).

12. I. Pascucci, D. Apai, Th. Henning, and C. P. Dullemond, Astrophys. J. Lett. 590, L111 (2003).

13. Ch. Walker, K. Wood, C. J. Lada, et al., Mon. Not. R. Astron. Soc. 351, 607 (2004).

14. N. I. Shakura and R. A. Syunyaev, Astron. Astrophys. 24, 337 (1973).

15. A. V. Tutukov and Ya. N. Pavlyuchenkov, Astron. Zh. 81, 655 (2004) [Astron. Rep. 48, 534 (2004)].

16. P. D’Alessio, J. Cantó, N. Calvet, and S. Lizano, Astrophys. J. 500, 411 (1998).

17. P. D'Alessio, N. Calvet, L. Hartmann, et al., Astrophys. J. 527, 893 (1999).

18. A. Dutrey, T. Henning, S. Guilloteau, et al., Astron. Astrophys. 464, 615 (2007).

19. G. Chabrier, I. Baraffe, F. Allard, and P. Hauschildt, Astrophys. J. 542, 464 (2000).

20. D. Semenov, D. Wiebe, and Th. Henning, Astron. Astrophys. 417, 93 (2004).

21. E. Bergin, N. Calvet, P. D’Alessio, and G. J. Herczeg, Astrophys. J. Lett., 591, L159 (2003).

22. A. E. Glassgold, E. D. Feigelson, T. Montmerle, and S. Wolk, ASP Conf. Ser. 341, 165 (2005).

23. T. J. Millar, P. R. A. Farquhar, and K. Willacy, Astron. Astrophys. Suppl. Ser. 121, 139 (1997).

24. D. Wiebe, D. Semenov, and Th. Henning, Astron. Astrophys. 399, 197 (2003).

25. Y. Aikawa, G. J. van Zadelhoff, E. F. van Dishoeck, and E. Herbst, Astron. Astrophys. 386, 622 (2002).

Translated by L. Yungel'son 